International Journal of Pure and Applied Mathematics

Volume 107 No. $4 \quad 2016,859-873$

ISSN: 1311-8080 (printed version); ISSN: 1314-3395 (on-line version)

url: http://www.ijpam.eu

doi: 10.12732/ijpam.v107i4.5

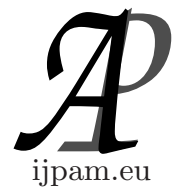

\title{
THE RELATIONS BETWEEN SOFT L-FUZZY TOPOGENOUS ORDERS AND SOFT L-FUZZY PRE-UNIFORMITIES
}

\author{
Yong Chan Kim \\ Department of Mathematics \\ Gangneung-Wonju University \\ Gangneung, Gangwondo 210-702, KOREA
}

\begin{abstract}
In this paper, we investigate the relations between soft $L$-fuzzy topogenous orders and soft $L$-fuzzy pre-uniform spaces. We give their examples.
\end{abstract}

AMS Subject Classification: 03E72, 06A15, 06F07, 54A05

Key Words: complete residuated lattice, soft $L$-quasi-uniformities, soft $L$-fuzzy topogenous orders

\section{Introduction}

Recently, Molodtsov [12] introduced the soft set as a mathematical tool for dealing information as the uncertainty of data in engineering, physics, computer sciences and many other diverse field. Presently, the soft set theory is making progress rapidly $[1,3]$. Pawlak's rough set $[13,14]$ can be viewed as a special case of soft rough sets [3]. The topological structures of soft sets have been developed by many researchers [2,7-10,15-18].

On the other hand, Hájek [4] introduced a complete residuated lattice which is an algebraic structure for many valued logic. It is an important mathematical tool for algebraic structures [5,7-10]. Kim [8] introduced a fuzzy soft $F: A \rightarrow$ $L^{U}$ as an extension as the soft $F: A \rightarrow P(U)$ where $L$ is a complete residuated lattice. Kim [7-10] introduced the soft topological structures, $L$-fuzzy quasiuniformities and soft $L$-fuzzy topogenous orders in complete residuated lattices.

Received: January 8, 2016

Published: May 5, 2016 (c) 2016 Academic Publications, Ltd.

url: www.acadpubl.eu 
In this paper, we investigate the relations between soft $L$-fuzzy topogenous orders and soft $L$-fuzzy pre-uniform spaces. We give their examples.

\section{Preliminaries}

Definition 1. [4,5] An algebra $(L, \wedge, \vee, \odot, \rightarrow, 0,1)$ is called a complete residuated lattice if it satisfies the following conditions:

(C1) $L=(L, \leq, \vee, \wedge, 1,0)$ is a complete lattice with the greatest element 1 and the least element 0 ;

(C2) $(L, \odot, 1)$ is a commutative monoid;

(C3) $x \odot y \leq z$ iff $x \leq y \rightarrow z$ for $x, y, z \in L$.

In this paper, we assume that $\left(L, \leq, \odot, \rightarrow, \oplus,{ }^{*}\right)$ is a complete residuated lattice with an order reversing involution * which is defined by $x \oplus y=\left(x^{*} \odot y^{*}\right)^{*}$ and $x^{*}=x \rightarrow 0$.

Lemma 2. [4,5] For each $x, y, z, x_{i}, y_{i}, w \in L$, we have the following properties:

(1) $1 \rightarrow x=x, 0 \odot x=0$,

(2) If $y \leq z$, then $x \odot y \leq x \odot z, x \oplus y \leq x \oplus z, x \rightarrow y \leq x \rightarrow z$ and $z \rightarrow x \leq y \rightarrow x$,

(3) $x \odot y \leq x \wedge y \leq x \vee y \leq x \oplus y$,

(4) $\left(\bigwedge_{i} y_{i}\right)^{*}=\bigvee_{i} y_{i}^{*},\left(\bigvee_{i} y_{i}\right)^{*}=\bigwedge_{i} y_{i}^{*}$,

(5) $x \odot\left(\bigvee_{i} y_{i}\right)=\bigvee_{i}\left(x \odot y_{i}\right)$,

(6) $x \oplus\left(\bigwedge_{i} y_{i}\right)=\bigwedge_{i}\left(x \oplus y_{i}\right)$,

(7) $x \rightarrow\left(\bigwedge_{i} y_{i}\right)=\bigwedge_{i}\left(x \rightarrow y_{i}\right)$,

(8) $\left(\bigvee_{i} x_{i}\right) \rightarrow y=\bigwedge_{i}\left(x_{i} \rightarrow y\right)$,

(9) $x \rightarrow\left(\bigvee_{i} y_{i}\right) \geq \bigvee_{i}\left(x \rightarrow y_{i}\right)$,

(10) $\left(\bigwedge_{i} x_{i}\right) \rightarrow y \geq \bigvee_{i}\left(x_{i} \rightarrow y\right)$,

(11) $(x \odot y) \rightarrow z=x \rightarrow(y \rightarrow z)=y \rightarrow(x \rightarrow z)$,

(12) $x \odot(x \rightarrow y) \leq y$ and $x \rightarrow y \leq(y \rightarrow z) \rightarrow(x \rightarrow z)$,

(13) $(x \rightarrow y) \odot(z \rightarrow w) \leq(x \odot z) \rightarrow(y \odot w)$, 
(14) $(x \rightarrow y) \odot(z \rightarrow w) \leq(x \oplus z) \rightarrow(y \oplus w)$,

$(15) x \rightarrow y \leq(x \odot z) \rightarrow(y \odot z)$ and $(x \rightarrow y) \odot(y \rightarrow z) \leq x \rightarrow z$,

(16) $x \odot y \odot(z \odot w) \leq(x \odot z) \oplus(y \odot w)$.

(17) $x \rightarrow y=y^{*} \rightarrow x^{*}$.

Definition 3. [7-10] Let $X$ be an initial universe of objects and $E$ the set of parameters (attributes) in $X$. A pair $(F, A)$ is called a fuzzy soft set over $X$, where $A \subset E$ and $F: A \rightarrow L^{X}$ is a mapping. We denote $S(X, A)$ as the family of all fuzzy soft sets under the parameter $A$.

Definition 4. [7-10] Let $(F, A)$ and $(G, A)$ be two fuzzy soft sets over a common universe $X$ :

(1) $(F, A)$ is a fuzzy soft subset of $(G, A)$, denoted by $(F, A) \leq(G, A)$ if $F(\epsilon) \leq G(\epsilon)$, for each $\epsilon \in A$.

(2) $(F, A) \wedge(G, A)=(F \wedge G, A)$ if $(F \wedge G)(\epsilon)=F(\epsilon) \wedge G(\epsilon)$ for each $\epsilon \in A$.

(3) $(F, A) \vee(G, A)=(F \vee G, A)$ if $(F \vee G)(\epsilon)=F(\epsilon) \vee G(\epsilon)$ for each $\epsilon \in A$.

(4) $(F, A) \odot(G, A)=(F \odot G, A)$ if $(F \odot G)(\epsilon)=F(\epsilon) \odot G(\epsilon)$ for each $\epsilon \in A$.

(5) $(F, A)^{*}=\left(F^{*}, A\right)$ if $F^{*}(\epsilon)=(F(\epsilon))^{*}$ for each $\epsilon \in A$.

(6) $(F, A) \oplus(G, A)=(F \oplus G, A)$ if $(F \oplus G)(\epsilon)=\left(F^{*}(\epsilon) \odot G^{*}(\epsilon)\right)^{*}$ for each $\epsilon \in A$.

Definition 5. [10] A mapping $\xi: S(X, A) \times S(X, A) \rightarrow L$ is called a soft $L$-fuzzy semi-topogenous order on $(X, A)$ if it satisfies the following axioms:

$(\mathrm{ST} 1) \xi\left(\left(1_{X}, A\right),\left(1_{X}, A\right)\right)=\xi\left(\left(0_{X}, A\right),\left(0_{X}, A\right)\right)=1$.

(ST2) If $\xi((F, A),(G, A)) \neq 0$, then $(F, A) \leq(G, A)$.

(ST3) If $\left(F_{1}, A\right) \leq(F, A), \quad(G, A) \leq\left(G_{1}, A\right)$, then $\xi((F, A),(G, A)) \leq$ $\xi\left(\left(F_{1}, A\right),\left(G_{1}, A\right)\right)$.

A mapping $\xi$ is called a soft strong $L$-fuzzy semi-topogenous order on $(X, A)$ if it satisfies (ST1), (ST3) and the following axiom.

(S) $\xi((F, A),(G, A)) \leq S((F, A),(G, A))$ where

$$
S((F, A),(G, A))=\bigwedge_{a \in A} \bigwedge_{x \in X}(F(a)(x) \rightarrow G(a)(x)) .
$$


Remark 6. If $\xi$ is a soft (resp. strong) $L$-fuzzy semi-topogenous order on $(X, A)$. Define a mapping $\xi^{s}: S(X, A) \times S(X, A) \rightarrow L$ as $\xi^{s}((F, A),(G, A))=$ $\xi\left((G, A)^{*},(F, A)^{*}\right)$. Then $\xi^{s}$ is a soft (resp. strong) $L$-fuzzy semi-topogenous order on $(X, A)$.

Definition 7. [10] A soft (resp. strong) $L$-fuzzy semi-topogenous order $\xi$ is called:

(1) soft (resp. strong) $L$-fuzzy topogenous if (T)

$\xi\left(\left(F_{1}, A\right) \odot\left(F_{2}, A\right),\left(G_{1}, A\right) \odot\left(G_{2}, A\right)\right) \geq \xi\left(\left(F_{1}, A\right),\left(G_{1}, A\right)\right) \odot \xi\left(\left(F_{2}, A\right),\left(G_{2}, A\right)\right)$.

(2) soft (resp. strong) L-fuzzy cotopogenous if (CT)

$\xi\left(\left(F_{1}, A\right) \oplus\left(F_{2}, A\right),\left(G_{1}, A\right) \oplus\left(G_{2}, A\right)\right) \geq \xi\left(\left(F_{1}, A\right),\left(G_{1}, A\right)\right) \odot \xi\left(\left(F_{2}, A\right),\left(G_{2}, A\right)\right)$,

(3) soft (resp. strong) $L$-fuzzy bitopogenous if $\xi$ are soft (resp. strong) $L$-fuzzy topogenous and soft (resp. strong) $L$-fuzzy cotopogenous.

Definition 8. [10] A soft (resp. strong) L-fuzzy topogenous (resp. cotopogenous) order $\xi$ on $(X, A)$ is said to be a soft (resp. strong) $L$-fuzzy topogenous (resp. cotopogenous) structure if $\xi \circ \xi \geq \xi$, where

$$
\begin{aligned}
& (\xi \circ \xi)((F, A),(H, A)) \\
& =\bigvee_{(G, A) \in S(X, A)} \xi((F, A),(G, A)) \odot \xi((G, A),(H, A))
\end{aligned}
$$

Definition 9. [10] A mapping $\mathcal{U}: S(X \times X, A) \rightarrow L$ is called a soft $L$-fuzzy pre-uniformity on $X$ iff it satisfies the properties:

(SU1) There exists $(U, A) \in S(X \times X, A)$ such that $\mathcal{U}((U, A))=1$,

(SU2) If $(V, A) \leq(U, A)$, then $\mathcal{U}((V, A)) \leq \mathcal{U}((U, A))$,

(SU3) For every $(U, A),(V, A) \in S(X \times X, A)$,

$$
\mathcal{U}((U, A) \odot(V, A)) \geq \mathcal{U}((U, A)) \odot \mathcal{U}((V, A))
$$

(SU4) If $\mathcal{U}((U, A)) \neq 0$, then $\left(1_{\triangle}, A\right) \leq(U, A)$, where

$$
1_{\Delta}(x, y)= \begin{cases}1 & \text { if } x=y \\ 0 & \text { if } x \neq y\end{cases}
$$


A soft $L$-fuzzy pre-uniformity $\mathcal{U}$ is called a soft $L$-fuzzy quasi-uniformity if

$$
\text { (Q) } \mathcal{U}(U, A) \leq \bigvee\{\mathcal{U}((V, A)) \odot \mathcal{U}((W, A)) \mid(V, A) \circ(W, A) \leq(U, A)\}
$$

where, for all $x, y \in X, a \in A$,

$$
(V(a) \circ W(a))(x, y)=\bigvee_{z \in X}(V(a)(z, x) \odot W(a)(x, y))
$$

The triple $(X, A, \mathcal{U})$ is called a soft $L$-fuzzy pre-(resp. quasi-) uniform space.

Remark 10. Let $(X, \mathcal{U})$ be a soft $L$-fuzzy quasi-uniform space, then by (SU1) and (SU2), we have $\mathcal{U}\left(1_{X \times X}\right)=1$ because $(U, A) \leq\left(1_{X \times X}, A\right)$ for all $(U, A) \in S(X \times X, A)$.

Lemma 11. $[7,10]$ For every $(F, A),(G, A) \in S(X, A)$, we define

$$
\left(U_{F, G}, A\right),\left(U_{F, G}^{-1}, A\right) \in S(X \times X, A)
$$

by

$$
\begin{gathered}
U_{F, G}(a)(x, y)=F(a)(x) \rightarrow G(a)(y), \\
U_{F, G}^{-1}(a)(x, y)=U_{F, G}(a)(y, x),
\end{gathered}
$$

then we have the following statements:

(1) $\left(1_{X \times X}, A\right)=\left(U_{0_{X}, 0_{X}}, A\right)=\left(U_{1_{X}, 1_{X}}, A\right)$,

(2) If $\left(F_{1}, A\right) \leq\left(F_{2}, A\right)$ and $\left(G_{1}, A\right) \leq\left(G_{2}, A\right)$, then $\left(U_{F_{2}, G_{1}}, A\right) \leq\left(U_{F_{1}, G_{2}}, A\right)$,

(3) If $(F, A) \leq(G, A)$, then $\left(1_{\triangle}, A\right) \leq\left(U_{F, G}, A\right)$,

(4) For every $\left(U_{F, G}, A\right) \in S(X \times X, A)$ and $(H, A) \in S(X, A)$, we have $\left(U_{H, G}, A\right) \circ\left(U_{F, H}, A\right) \leq\left(U_{F, G}, A\right)$,

(5) $\left(U_{F_{1}, G_{1}}, A\right) \odot\left(U_{F_{2}, G_{2}}, A\right) \leq\left(U_{F_{1} \odot F_{2}, G_{1} \odot G_{2}}, A\right)$,

(6) $\left(U_{F_{1}, G_{1}}, A\right) \odot\left(U_{F_{2}, G_{2}}, A\right) \leq\left(U_{F_{1} \oplus F_{2}, G_{1} \oplus G_{2}}, A\right)$,

(7) $\left(U_{F, G}^{-1}, A\right)=\left(U_{G^{*}, F^{*}}, A\right)$,

(8) $\left(U_{F_{1}, \odot F_{2}, G_{1} \odot G_{2}}^{-1}, A\right)=\left(U_{G_{1}^{*} \oplus G_{2}^{*}, F_{1}^{*} \oplus F_{2}^{*}}, A\right)$,

(9) $\left(U_{F_{1} \oplus F_{2}, G_{1} \oplus G_{2}}^{-1}, A\right)=\left(U_{G_{1}^{*} \odot G_{2}^{*}, F_{1}^{*} \odot F_{2}^{*}}, A\right)$. 
Lemma 12. $[7,10]$ Let $(X, A, \mathcal{U})$ be a soft $L$-fuzzy quasi uniform space. For each $(U, A) \in S(X \times X, A)$ and $(F, A) \in S(X, A)$, the images $(U, A)[(F, A)]$, $(U, A)[[(F, A)]]$ of $(F, A)$ with respect to $(U, A)$ are defined by, for all $x \in X, a \in$ A,

$$
\begin{aligned}
(U, A)[(F, A)](a)(x) & =\bigvee_{y \in X}(F(a)(y) \odot U(a)(y, x)), \\
(U, A)[[(F, A)]](a)(x) & =\bigvee_{y \in X}(F(a)(y) \odot U(a)(x, y)) .
\end{aligned}
$$

For each

$$
(U, A),(V, A),\left(U_{1}, A\right),\left(U_{2}, A\right) \in S(X \times X, A)
$$

and

$$
(F, A),(G, A),\left(F_{1}, A\right),\left(F_{2}, A\right),\left(F_{i}, A\right) \in S(X, A),
$$

we have:

(1) $(F, A) \leq(U, A)[(F, A)]$ and $(F, A) \leq(U, A)[[(F, A)]]$ for each $\mathcal{U}((U, A))>0$

(2) $(U, A) \leq(U, A) \circ(U, A)$, for each $\mathcal{U}((U, A))>0$,

(3) $((V, A) \circ(U, A))[(F, A)]=(V, A)[(U, A)[(F, A)]]$,

$((V, A) \circ(U, A))[[(F, A)]]=(V, A)[[(U, A)[[(F, A)]]]]$,

(4) $(U, A)\left[\bigvee_{i}\left(F_{i}, A\right)\right]=\bigvee_{i}(U, A)\left[\left(F_{i}, A\right)\right]$ and $(U, A)\left[\left[\bigvee_{i}\left(F_{i}, A\right)\right]\right]=\bigvee_{i}(U, A)\left[\left[\left(F_{i}, A\right)\right]\right]$,

(5) $\left(\left(U_{1}, A\right) \odot\left(U_{2}, A\right)\right)\left[\left(F_{1}, A\right) \odot\left(F_{2}, A\right)\right] \leq\left(U_{1}, A\right)\left[\left(F_{1}, A\right)\right] \odot\left(U_{2}, A\right)\left[\left(F_{2}, A\right)\right]$,

(6) $\left(\left(U_{1}, A\right) \odot\left(U_{2}, A\right)\right)\left[\left[\left(F_{1}, A\right) \odot\left(F_{2}, A\right)\right]\right]$

$\leq\left(U_{1}, A\right)\left[\left[\left(F_{1}, A\right)\right]\right] \odot\left(U_{2}, A\right)\left[\left[\left(F_{2}, A\right)\right]\right]$,

(7) $\left(\left(U_{1}, A\right) \odot\left(\left(U_{2}, A\right), A\right)\right)\left[\left(F_{1}, A\right) \oplus\left(F_{2}, A\right)\right]$

$\leq\left(U_{1}, A\right)\left[\left(F_{1}, A\right)\right] \oplus\left(\left(U_{2}, A\right), A\right)\left[\left(F_{2}, A\right)\right]$,

(8) $\left(\left(U_{1}, A\right) \odot\left(\left(U_{2}, A\right), A\right)\right)\left[\left[\left(F_{1}, A\right) \oplus\left(F_{2}, A\right)\right]\right]$

$\leq\left(U_{1}, A\right)\left[\left[\left(F_{1}, A\right)\right]\right] \oplus\left(\left(U_{2}, A\right), A\right)\left[\left[\left(F_{2}, A\right)\right]\right]$.

(9) $\left(U_{F, G}, A\right)=\bigvee\{(W, A) \in S(X \times X, A) \mid(W, A)[(F, A)] \leq(G, A)\}$.

(10) $\left(U_{F, G}^{-1}, A\right)=\bigvee\{(W, A) \in S(X \times X, A) \mid(W, A)[[(F, A)]] \leq(G, A)\}$.

(11) $\left(U_{F, G}, A\right)[(F, A)] \leq(G, A)$ and $\left(U_{F, G}, A\right)\left[\left[(G, A)^{*}\right]\right] \leq(F, A)^{*}$. Moreover, $\left(U_{F, F}, A\right)[(F, A)]=(F, A)$ and $\left(U_{F, F}, A\right)\left[(F, A)^{*}\right]=(F, A)^{*}$. 
Theorem 13. [10] Let $(X, A, \mathcal{U})$ be a soft $L$-fuzzy quasi-uniform space. Define mappings $\xi_{\mathcal{U}}^{r}, \xi_{\mathcal{U}}^{l}: S(X, A) \times S(X, A) \rightarrow L$ by

$$
\begin{aligned}
& \xi_{\mathcal{U}}^{r}((F, A),(G, A))=\bigvee\{\mathcal{U}((U, A)) \mid(U, A)[(F, A)] \leq(G, A)\}, \\
& \xi_{\mathcal{U}}^{l}((F, A),(G, A))=\bigvee\{\mathcal{U}((U, A)) \mid(U, A)[[(F, A)]] \leq(G, A)\}
\end{aligned}
$$

Then $\xi_{\mathcal{U}}^{r}$ and $\xi_{\mathcal{U}}^{l}$ are soft L-fuzzy bitopogenous stuctures.

Theorem 14. [7] Let $\xi$ be a soft $L$-fuzzy topogenous order on $(X, A)$. Define $\mathcal{U}_{\xi}: S(X \times X, A) \rightarrow L$ by

$$
\mathcal{U}_{\xi}((U, A))=\bigvee\left\{\odot_{i=1}^{n} \xi\left(\left(F_{i}, A\right),\left(G_{i}, A\right)\right) \mid \odot_{i=1}^{n}\left(U_{F_{i}, G_{i}}, A\right) \leq(U, A)\right\},
$$

where $\bigvee$ is taken over every finite family $\left\{\left(U_{F_{i}, G_{i}}, A\right) \mid i=1,2,3, \ldots, n\right\}$. Then:

(1) $\mathcal{U}_{\xi}\left(\left(U_{\odot_{i=1}^{n}} F_{i}, \odot_{i=1}^{n} G_{i}, A\right)\right)=\xi\left(\odot_{i=1}^{n}\left(F_{i}, A\right), \odot_{i=1}^{n}\left(G_{i}, A\right)\right)$.

(2) $\mathcal{U}_{\xi}$ is a soft $L$-fuzzy pre-uniformity on $X$.

(3) If $\xi$ is a soft $L$-fuzzy topogenous structure on $(X, A)$, then $\mathcal{U}_{\xi}$ is a soft $L$-fuzzy quasi-uniformity on $X$.

(4) $\xi_{\mathcal{U}_{\xi}}^{r}=\xi$ and $\xi_{\mathcal{U}_{\xi}}^{l}=\xi^{s}$.

Theorem 15. [7] Let $\xi$ be a soft $L$-fuzzy cotopogenous order on $(X, A)$. Define $\mathcal{U}_{\xi}: S(X \times X, A) \rightarrow L$ by

$$
\begin{aligned}
\mathcal{U}_{\xi}((U, A)) & =\bigvee\left\{\odot_{i=1}^{n} \xi\left(\left(F_{i}, A\right),\left(G_{i}, A\right)\right) \mid\right. \\
& \left.\odot_{i=1}^{n}\left(U_{F_{i}, G_{i}}, A\right) \leq(U, A)\right\},
\end{aligned}
$$

where $\bigvee$ is taken over every finite family $\left\{\left(U_{F_{i}, G_{i}}, A\right) \mid i=1,2,3, \ldots, n\right\}$. Then:

(1) $\mathcal{U}_{\xi}\left(\left(U_{\oplus_{i=1}^{n} F_{i}, \oplus_{i=1}^{n} G_{i}}, A\right)\right)=\xi\left(\oplus_{i=1}^{n}\left(F_{i}, A\right), \oplus_{i=1}^{n}\left(G_{i}, A\right)\right)$.

(2) $\mathcal{U}_{\xi}$ is a soft $L$-fuzzy pre-uniformity on $X$.

(3) If $\xi$ is a soft $L$-fuzzy cotopogenous structure on $(X, A)$, then $\mathcal{U}_{\xi}$ is a soft $L$-fuzzy quasi-uniformity on $X$.

(4) $\mathcal{U}_{\xi^{s}}((U, A))=\mathcal{U}_{\xi}\left((U, A)^{-1}\right)$ for all $(U, A) \in S(X \times X, L)$.

(5) $\xi_{\mathcal{U}_{\xi}}^{r}=\xi$ and $\xi_{\mathcal{U}_{\xi}}^{l}=\xi^{s}$. 


\section{The Relations between Soft $L$-Fuzzy Topogenous Orders and Soft $L$-Fuzzy Pre-Uniformities}

Definition 16. A soft $L$-fuzzy pre-uniform structure $\mathcal{U}$ on $X$ is said to be right compatible (resp. left compatible) with a soft $L$-fuzzy topogenous order $\xi$ on $(X, A)$ if $\xi_{\mathcal{U}}^{r}=\xi$ (resp. $\left.\xi_{\mathcal{U}}^{l}=\xi\right)$.

The class $\Pi^{r}(\xi)$ (resp. $\Pi^{l}(\xi)$ ) denotes the family of all soft $L$-fuzzy preuniformities which are right compatible(resp. left compatible) with a given soft $L$-fuzzy topogenous structure $\xi$.

Theorem 17. Let $\xi$ be a soft $L$-fuzzy topogenous order on $(X, A)$ and the soft $L$-fuzzy topogenous orders $\xi_{\mathcal{U}_{\xi}}^{r}$ and $\xi_{\mathcal{U}_{\xi}}^{l}$ induced by $\mathcal{U}_{\xi}$. Then we have:

(1) $\xi_{\mathcal{U}_{\xi}}^{r}=\xi$, that is, $\mathcal{U}_{\xi} \in \Pi^{r}(\xi)$.

(2) $\xi_{\mathcal{U}_{\xi}}^{l}=\xi^{s}$, that is, $\mathcal{U}_{\xi} \in \Pi^{l}\left(\xi^{s}\right)$.

(3) $\mathcal{U}_{\xi}$ is the coarsest member of $\Pi^{r}(\xi)$;i.e. $\mathcal{U}_{\xi} \leq \mathcal{U}$ for all $\mathcal{U} \in \Pi^{r}(\xi)$.

(4) $\mathcal{U}_{\xi}$ is the coarsest member of $\Pi^{l}\left(\xi^{s}\right)$.

Proof (1) and (2)are easily proved from Theorems 14 (4) and 15 (5).

(3) By (1), we have that $\mathcal{U}_{\xi}$ is right compatible with $\xi$. Let $\mathcal{U}$ be an arbitrary member of $\Pi^{r}(\xi)$. We will show that $\mathcal{U}_{\xi}((U, A)) \leq \mathcal{U}((U, A))$, for all $(U, A) \in$ $S(X \times X, A)$.

Suppose that there exists $(U, A) \in S(X \times X, A)$ such that

$$
\mathcal{U}_{\xi}((U, A)) \not \leq \mathcal{U}((U, A)) \text {. }
$$

There exists a finite family $\left\{\left(U_{F_{i}, G_{i}}, A\right) \mid \odot_{i=1}^{m}\left(U_{F_{i}, G_{i}}, A\right) \leq(U, A)\right\}$ such that

$$
\odot_{i=1}^{m} \xi\left(\left(F_{i}, A\right),\left(G_{i}, A\right)\right) \not \leq \mathcal{U}((U, A)) \text {. }
$$

Since $\mathcal{U} \in \Pi^{r}(\xi)$, that is, $\xi\left(\lambda_{i}, \rho_{i}\right)=\xi_{\mathcal{U}}^{r}\left(\lambda_{i}, \rho_{i}\right)$ for $i=1, \ldots, m$ and $L$ is a complete residuated lattice, by the definition of $\xi_{\mathcal{U}}^{r}$, there exists $\left(V_{i}, A\right) \in S(X \times X, A)$ with $\left(V_{i}, A\right)\left[\left(F_{i}, A\right)\right] \leq\left(G_{i}, A\right)$ such that

$$
\odot_{i=1}^{m} \mathcal{U}\left(\left(V_{i}, A\right)\right) \not \leq \mathcal{U}((U, A)) .
$$

On the other hand, put $(V, A)=\odot_{i=1}^{m}\left(V_{i}, A\right)$. Since $\left(V_{i}, A\right)\left[\left(F_{i}, A\right)\right] \leq\left(G_{i}, A\right)$, by the definition of $\left(U_{F_{i}, G_{i}}, A\right)$, we have $\left(V_{i}, A\right) \leq\left(U_{F_{i}, G_{i}}, A\right)$. It follows that

$$
(V, A)=\odot_{i=1}^{m}\left(V_{i}, A\right) \leq \odot_{i=1}^{m}\left(U_{F_{i}, G_{i}}, A\right) \leq(U, A)
$$

Hence

$$
\begin{aligned}
& \odot_{i=1}^{m} \mathcal{U}\left(V_{i}, A\right) \leq \mathcal{U}(V, A) \\
& \leq \mathcal{U}\left(\odot_{i=1}^{m}\left(U_{F_{i}, G_{i}}, A\right)\right) \leq \mathcal{U}((U, A)) .
\end{aligned}
$$


It is a contradiction for the equation (I).

(4) It is similarly proved as (3).

Theorem 18. Let $(X, A, \mathcal{U})$ be a soft $L$-fuzzy pre-uniform space. Then:

(1) $\mathcal{U}_{\xi_{\mathcal{U}}}((U, A)) \leq \mathcal{U}\left((U, A)^{-1}\right)$ for all $(U, A) \in S(X \times X, A)$.

(2) $\mathcal{U}_{\xi_{\mathcal{U}}^{r}}((U, A)) \leq \mathcal{U}((U, A))$ for all $(U, A) \in S(X \times X, A)$.

Proof (1) Suppose that there exists $(U, A) \in S(X \times X, A)$ such that

$$
\mathcal{U}_{\xi_{\mathcal{U}}^{l}}((U, A)) \not \subset \mathcal{U}\left((U, A)^{-1}\right) \text {. }
$$

From the definition of $\mathcal{U}_{\xi_{\mathcal{U}}^{l}}$, there exists a finite family $\left\{\left(U_{F_{i}, G_{i}}, A\right) \mid \odot_{i=1}^{n}\left(U_{F_{i}, G_{i}}, A\right) \leq\right.$ $(U, A)\}$ such that

$$
\odot_{i=1}^{n} \xi_{\mathcal{U}}^{l}\left(\left(F_{i}, A\right),\left(G_{i}, A\right)\right) \not \leq \mathcal{U}\left((U, A)^{-1}\right) .
$$

From the definition of $\xi_{\mathcal{U}}^{l}$, for each $i \in\{1,2, \ldots, n\}$, there exists $\left(V_{i}, A\right) \in S(X \times$ $X, A)$ with $\left(V_{i}, A\right)\left[\left[\left(F_{i}, A\right)\right]\right] \leq\left(G_{i}, A\right)$ such that

$$
\odot_{i=1}^{n} \mathcal{U}\left(\left(V_{i}, A\right)\right) \not \leq \mathcal{U}\left(\left(U^{-1}, A\right)\right) \text {. }
$$

Let $(V, A)=\odot_{i=1}^{n}\left(V_{i}, A\right)$ be given. Since $\left(V_{i}, A\right)\left[\left[\left(F_{i}, A\right)\right]\right] \leq\left(G_{i}, A\right)$, by the definition of $\left(U_{F_{i}, G_{i}}^{-1}, A\right)$, we have $\left(V_{i}, A\right) \leq\left(U_{F_{i}, G_{i}}^{-1}, A\right)$. Hence $\left(U_{F_{i}, G_{i}}^{-1}, A\right) \geq$ $\odot_{i=1}^{n}\left(U_{F_{i}, G_{i}}^{-1}, A\right) \geq \odot_{i=1}^{n}\left(V_{i}, A\right)=(V, A)$ and

$$
\mathcal{U}\left(\left(U^{-1}, A\right)\right) \geq \mathcal{U}\left(\odot_{i=1}^{n}\left(U_{F_{i}, G_{i}}^{-1}, A\right)\right) \geq \odot_{i=1}^{n} \mathcal{U}\left(\left(V_{i}, A\right)\right) .
$$

It is a contradiction. Therefore, $\mathcal{U}_{\xi_{\mathcal{U}}^{l}}((U, A)) \leq \mathcal{U}\left(\left(U^{-1}, A\right)\right)$.

(2) It is similarly proved as (1).

Definition 19. [10] Let $S(X, A)$ and $S(Y, B)$ be the families of all fuzzy soft sets over $X$ and $Y$, respectively. The mapping $f_{\phi}: S(X, A) \rightarrow S(Y, B)$ is a soft mapping where $f: X \rightarrow Y$ and $\phi: A \rightarrow B$ are mappings.

(1) The image of $(F, A) \in S(X, A)$ under the mapping $f_{\phi}$ is denoted by $f_{\phi}((F, A))=\left(f_{\phi}(F), B\right)$ where

$$
f_{\phi}(F)(b)= \begin{cases}\bigvee_{a \in \phi^{-1}(\{b\})} f^{\rightarrow}(F(a)), & \text { if } \phi^{-1}(\{b\}) \neq \emptyset \\ 0, & \text { otherwise. }\end{cases}
$$

(2) The inverse image of $(G, B) \in S(Y, B)$ under the mapping $f_{\phi}$ is denoted by $f_{\phi}^{-1}((G, B))=\left(f_{\phi}^{-1}(G), A\right)$ where

$$
f_{\phi}^{-1}(G)(a)(x)=f^{\leftarrow}(G(\phi(a)))(x), \forall a \in A, x \in X .
$$


(3) The soft mapping $f_{\phi}: S(X, A) \rightarrow S(Y, B)$ is called injective (resp. surjective, bijective) if $f$ and $\phi$ are both injective (resp. surjective, bijective).

Lemma 20. [10] Let $f_{\phi}: S(X, A) \rightarrow S(Y, B)$ be a soft mapping. Then we have the following properties. For $(F, A),\left(F_{i}, A\right) \in S(X, A)$ and $(G, B),\left(G_{i}, B\right) \in$ $S(Y, B)$,

(1) $(G, B) \geq f_{\phi}\left(f_{\phi}^{-1}((G, B))\right)$ with equality if $f$ is surjective,

(2) $(F, A) \leq f_{\phi}^{-1}\left(f_{\phi}((F, A))\right)$ with equality if $f$ is injective,

(3) $f_{\phi}^{-1}\left((G, B)^{*}\right)=\left(f_{\phi}^{-1}((G, B))\right)^{*}$,

(4) $f_{\phi}^{-1}\left(\bigvee_{i \in I}\left(G_{i}, B\right)\right)=\bigvee_{i \in I} f_{\phi}^{-1}\left(\left(G_{i}, B\right)\right)$,

(5) $f_{\phi}^{-1}\left(\bigwedge_{i \in I}\left(G_{i}, B\right)\right)=\bigwedge_{i \in I} f_{\phi}^{-1}\left(\left(G_{i}, B\right)\right)$,

(6) $f_{\phi}\left(\bigvee_{i \in I}\left(F_{i}, A\right)\right)=\bigvee_{i \in I} f_{\phi}\left(\left(F_{i}, A\right)\right)$,

(7) $f_{\phi}\left(\bigwedge_{i \in I}\left(F_{i}, A\right)\right) \leq \bigwedge_{i \in I} f_{\phi}\left(\left(F_{i}, A\right)\right)$ with equality if $f$ is injective,

(8) $f_{\phi}^{-1}\left(\left(G_{1}, B\right) \odot\left(G_{2}, B\right)\right)=f_{\phi}^{-1}\left(\left(G_{1}, B\right)\right) \odot f_{\phi}^{-1}\left(\left(G_{2}, B\right)\right)$,

(9) $f_{\phi}^{-1}\left(\left(G_{1}, B\right) \oplus\left(G_{2}, B\right)\right)=f_{\phi}^{-1}\left(\left(G_{1}, B\right)\right) \oplus f_{\phi}^{-1}\left(\left(G_{2}, B\right)\right)$,

(10) $f_{\phi}\left(\left(F_{1}, A\right) \odot\left(F_{2}, A\right)\right) \leq f_{\phi}\left(\left(F_{1}, A\right)\right) \odot f_{\phi}\left(\left(F_{2}, A\right)\right)$ with equality if $f$ is injective,

(11) $f_{\phi}\left(\left(F_{1}, A\right) \oplus\left(F_{2}, A\right)\right) \leq f_{\phi}\left(\left(F_{1}, A\right)\right) \oplus f_{\phi}\left(\left(F_{2}, A\right)\right)$ with equality if $f$ is injective.

Theorem 21. [10] Let $(X, A, \mathcal{U})$ and $(Y, B, \mathcal{V})$ be soft $L$-fuzzy quasiuniform spaces. Let $f_{\phi}:(X, A, \mathcal{U}) \rightarrow(Y, B, \mathcal{V})$ be a uniformly continuous soft map. Then:

(1) $f_{\phi}:\left(X, A, \xi_{\mathcal{U}}^{r}\right) \rightarrow\left(Y, B, \xi_{\mathcal{V}}^{r}\right)$ is a topogenous continuous soft map.

(2) $f_{\phi}:\left(X, A, \xi_{\mathcal{U}}^{l}\right) \rightarrow\left(Y, B, \xi_{\mathcal{V}}^{l}\right)$ is a topogenous continuous soft map.

Theorem 22. Let $\left(X, A, \xi_{X}\right)$ and $\left(Y, B, \xi_{Y}\right)$ be soft $L$-fuzzy topogenous ordered sets. Then a mapping $f_{\phi}:\left(X, \xi_{X}, A\right) \rightarrow\left(Y, \xi_{Y}, B\right)$ is a topogenous continuous soft map iff the mapping $f_{\phi}:\left(X, A, \mathcal{U}_{\xi_{X}}\right) \rightarrow\left(Y, A, \mathcal{U}_{\xi_{Y}}\right)$ is an uniformly continuous soft map.

Proof. Since $f_{\phi}:\left(X, \xi_{X}, A\right) \rightarrow\left(Y, \xi_{Y}, B\right)$ is a topogenous continuous soft map, then

$$
\begin{aligned}
& (f \times f)_{\phi}^{-1}\left(\left(U_{F, G}, B\right)\right)(a)(x, y)=U_{F, G}(\phi(a))(f(x), f(y)) \\
& =F(\phi(a))(f(x)) \rightarrow G(\phi(a))(f(y))=f_{\phi}^{-1}(F)(a)(x) \rightarrow f_{\phi}^{-1}(G)(a)(y) \\
& =\left(U_{f_{\phi}^{-1}(F), f_{\phi}^{-1}(G)}, A\right)(a)(x, y)
\end{aligned}
$$




$$
\begin{aligned}
& \mathcal{U}_{\xi_{X}}\left((f \times f)_{\phi}^{-1}((V, B))\right) \\
& \geq \bigvee\left\{\odot_{i=1}^{n} \xi_{X}\left(f_{\phi}^{-1}\left(\left(F_{i}, B\right)\right), f_{\phi}^{-1}\left(\left(G_{i}, B\right)\right)\right) \mid\right. \\
& \left.\odot_{i=1}^{n}\left(U_{f_{\phi}^{-1}\left(F_{i}\right), f_{\phi}^{-1}\left(G_{i}\right)}, A\right) \leq(f \times f)_{\phi}^{-1}((V, B))\right\} \\
& \geq \bigvee\left\{\odot_{i=1}^{n} \xi_{Y}\left(\left(F_{i}, B\right),\left(G_{i}, B\right)\right) \mid \odot_{i=1}^{n}\left(\left(U_{F_{i}, G_{i}}, B\right)\right) \leq(V, B)\right\} \\
& =\mathcal{U}_{\xi_{Y}}((V, B)) .
\end{aligned}
$$

Conversely, let $f:\left(X, \mathcal{U}_{\xi_{X}}\right) \rightarrow\left(Y, \mathcal{U}_{\xi_{Y}}\right)$ is an uniformly continuous soft map. Since $\xi_{\mathcal{U}_{\xi_{X}}}^{r}=\xi_{X}$ and $\xi_{\mathcal{U}_{\xi_{Y}}}^{r}=\xi_{Y}$, by Theorems 15(4) and 21, the result holds.

Example 23. Let $U=\left\{h_{i} \mid i=\{1, \ldots, 6\}\right\}$ with $h_{i}=$ house and $E=$ $\{e, b, w, c, i\}$ with $e=$ expensive, $b=$ beautiful, $w=$ wooden, $c=$ creative, $i=$ in the green surroundings.

Define a binary operation $\odot$ on $[0,1]$ by

$$
\begin{gathered}
x \odot y=\max \{0, x+y-1\}, x \rightarrow y=\min \{1-x+y, 1\} \\
x \oplus y=\min \{1, x+y\}, x^{*}=1-x
\end{gathered}
$$

Then $([0,1], \wedge, \rightarrow, 0,1)$ is a complete residuated lattice (ref.[4.5]). Let $A=$ $\{b, c, i\} \subset E$ and $X=\left\{h_{1}, h_{4}, h_{5}, h_{6}\right\}$. Put $(H, A)$ be a fuzzy soft set as follow:

$$
\begin{array}{cccccc}
(H, A) & h_{1} & h_{4} & h_{5} & h_{6} & \\
b & 0.5 & 0.6 & 0.2 & 0.6 & \\
c & 0.1 & 0.5 & 0.5 & 0.6 & \\
i & 0.4 & 0.6 & 0.6 & 0.5 & \\
(H, A) \odot(H, A) & h_{1} & h_{4} & h_{5} & h_{6} \\
b & & 0.0 & 0.2 & 0.0 & 0.2 \\
c & & 0.0 & 0.0 & 0.0 & 0.2 \\
i & & 0.0 & 0.2 & 0.2 & 0.0 \\
\left(H^{*}, A\right) & h_{1} & h_{4} & h_{5} & h_{6} & \\
b & 0.5 & 0.4 & 0.8 & 0.4 & \\
c & 0.9 & 0.5 & 0.5 & 0.4 & \\
i & 0.6 & 0.4 & 0.4 & 0.5 & \\
\left(H^{*}, A\right) \oplus\left(H^{*}, A\right) & h_{1} & h_{4} & h_{5} & h_{6} \\
b & & 1.0 & 0.8 & 1.0 & 0.8 \\
c & & 1.0 & 1.0 & 1.0 & 0.8 \\
i & & 1.0 & 0.8 & 0.8 & 1.0
\end{array}
$$


(1) Define a soft $L$-fuzzy topogenous order $\xi: S(X, A) \times S(X, A) \rightarrow L$ as follows

$$
\xi((F, A),(G, A))= \begin{cases}1, & \text { if }(F, A)=(\overline{0}, A) \text { or }(G, A)=(\overline{1}, A) \\ 0.6, & \text { if }(F, A) \leq(H, A) \leq(G, A), \\ & (F, A) \not{L}(H, A) \odot(H, A) \\ 0.3, \quad \text { if }(\overline{0}, A) \neq(F, A) \leq(H, A) \odot(H, A) & \leq(G, A),(H, A) \not \leq(G, A), \\ 0, & \text { otherwise, }\end{cases}
$$

We obtain $\left(U_{H, H}, A\right),\left(U_{H \odot H, H \odot H}, A\right) \in S(X \times X, A)$ such that, for $a \in A$, $U_{H, H}(a) \in L^{X \times X}$ with $U_{H, H}(a)(x, y)=H(a)(x) \rightarrow H(a)(y)$,

$$
\begin{aligned}
& U_{H, H}(b)=\left(\begin{array}{cccc}
1 & 1 & 0.7 & 1 \\
0.9 & 1 & 0.6 & 1 \\
1 & 1 & 1 & 1 \\
0.9 & 1 & 0.6 & 1
\end{array}\right), U_{H, H}(c)=\left(\begin{array}{cccc}
1 & 1 & 1 & 1 \\
0.6 & 1 & 1 & 1 \\
0.6 & 1 & 1 & 1 \\
0.5 & 0.9 & 0.9 & 1
\end{array}\right) \\
& U_{H, H}(i)=\left(\begin{array}{cccc}
1 & 1 & 1 & 1 \\
0.8 & 1 & 1 & 0.9 \\
0.8 & 1 & 1 & 0.9 \\
0.9 & 1 & 1 & 1
\end{array}\right), U_{H \odot H, H \odot H}(b)=\left(\begin{array}{cccc}
1 & 1 & 1 & 1 \\
0.8 & 1 & 0.8 & 1 \\
1 & 1 & 1 & 1 \\
0.8 & 1 & 0.8 & 1
\end{array}\right) \\
& U_{H \odot H, H \odot H}(c)=\left(\begin{array}{cccc}
1 & 1 & 1 & 1 \\
1 & 1 & 1 & 1 \\
1 & 1 & 1 & 1 \\
0.8 & 0.8 & 0.8 & 1
\end{array}\right), U_{H \odot H, H \odot H}(i)=\left(\begin{array}{cccc}
1 & 1 & 1 & 1 \\
0.8 & 1 & 1 & 0.8 \\
0.8 & 1 & 1 & 0.8 \\
1 & 1 & 1 & 1
\end{array}\right)
\end{aligned}
$$

By Theorem 14, we obtain a soft $L$-fuzzy pre-uniformity $\mathcal{U}_{\xi}: S(X \times X, A) \rightarrow$ $L$ as follows

$$
\mathcal{U}_{\xi}((U, A))= \begin{cases}1, & \text { if }(U, A)=\left(1_{X \times X}, A\right), \\ 0.6, & \text { if }\left(U_{H, H}, A\right) \leq(U, A) \neq\left(1_{X \times X}, A\right), \\ 0.3, & \text { if }\left(U_{H \odot H, H \odot H}, A\right) \leq(U, A) \\ & (U, A) \geq\left(U_{H, H}, A\right), \\ 0.2, & \text { if }\left(U_{H, H}, A\right) \odot\left(U_{H, H}, A\right) \leq(U, A), \\ & (U, A) \geq\left(U_{H \odot H, H \odot H}, A\right), \\ 0, & \text { otherwise. }\end{cases}
$$

Since $U_{H, H}(H, A)=(H, A)$ and $\left(U_{H \odot H, H \odot H}, A\right)(H \odot H, A)=(H \odot H, A)$ from Lemma 12 (11), by Theorems 13 and 14(4), we have $\xi_{\mathcal{U}_{\xi}}^{r}=\xi$, that is, $\mathcal{U}_{\xi} \in \Pi^{r}(\xi)$. 
(2) By Remark 6 and (1), we obtain a soft $L$-fuzzy cotopogenous order $\xi^{s}: S(X, A) \times S(X, A) \rightarrow L$ as follows

$$
\xi^{s}((F, A),(G, A))= \begin{cases}1, & \text { if }(F, A)=(\overline{0}, A) \text { or }(G, A)=(\overline{1}, A) \\ 0.6, & \text { if }(F, A) \leq(H, A)^{*} \leq(G, A), \\ & (G, A) \ngtr(H, A)^{*} \oplus(H, A)^{*} \\ 0.3, & \text { if }(F, A) \leq(H, A)^{*} \oplus(H, A)^{*} \\ & \leq(G, A) \neq(\overline{1}, A),(F, A) \not \leq(H, A)^{*}, \\ 0, & \text { otherwise, }\end{cases}
$$

We obtain $\left(U_{H, H}, A\right),\left(U_{H \odot H, H \odot H}, A\right) \in S(X \times X, A)$ such that, for $a \in A$, $U_{H, H}(a) \in L^{X \times X}$ with $U_{H, H}(a)(x, y)=H(a)(x) \rightarrow H(a)(y)$,

$$
\begin{aligned}
& U_{H^{*}, H^{*}}(b)=\left(\begin{array}{cccc}
1 & 0.9 & 1 & 0.9 \\
1 & 1 & 1 & 1 \\
0.7 & 0.6 & 1 & 0.6 \\
1 & 1 & 1 & 1
\end{array}\right), U_{H^{*}, H^{*}}(c)=\left(\begin{array}{cccc}
1 & 0.6 & 0.6 & 0.5 \\
1 & 1 & 1 & 0.9 \\
1 & 1 & 1 & 0.9 \\
1 & 1 & 1 & 1
\end{array}\right) \\
& U_{H^{*}, H^{*}}(i)=\left(\begin{array}{cccc}
1 & 0.8 & 0.8 & 0.9 \\
1 & 1 & 1 & 1 \\
1 & 1 & 1 & 1 \\
1 & 0.9 & 0.9 & 1
\end{array}\right), U_{H^{*} \oplus H^{*}, H^{*} \oplus H^{*}}(b)=\left(\begin{array}{cccc}
1 & 0.8 & 1 & 0.8 \\
1 & 1 & 1 & 1 \\
1 & 0.8 & 1 & 0.8 \\
1 & 1 & 1 & 1
\end{array}\right) \\
& U_{H^{*} \oplus H^{*}, H^{*} \oplus H^{*}}(c)=\left(\begin{array}{cccc}
1 & 1 & 1 & 0.8 \\
1 & 1 & 1 & 0.8 \\
1 & 1 & 1 & 0.8 \\
1 & 1 & 1 & 1
\end{array}\right), U_{H^{*} \oplus H^{*}, H^{*} \oplus H^{*}}(i)=\left(\begin{array}{cccc}
1 & 0.8 & 0.8 & 1 \\
1 & 1 & 1 & 1 \\
1 & 1 & 1 & 1 \\
1 & 0.8 & 0.8 & 1
\end{array}\right)
\end{aligned}
$$

By Theorem 15, we obtain a soft $L$-fuzzy pre-uniformity $\mathcal{U}_{\xi^{s}}: S(X \times$ $X, A) \rightarrow L$ as follows

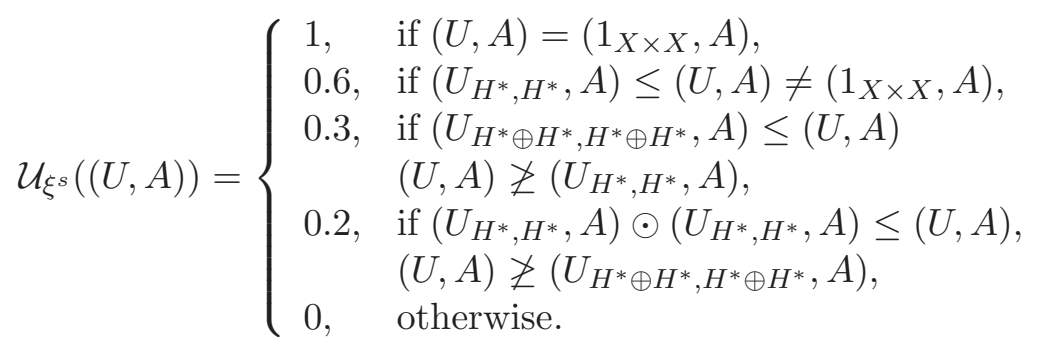

Since $U_{H^{*}, H^{*}}\left(H^{*}, A\right)=\left(H^{*}, A\right)$ and $\left(U_{H^{*} \oplus H^{*}, H^{*} \oplus H^{*}}, A\right)\left(H^{*} \oplus H^{*}, A\right)=\left(H^{*} \oplus\right.$ $H^{*}, A$ ) from Lemma $12(11)$, by Theorems 13 and $15(5)$, we have $\xi_{\mathcal{U}_{\xi^{s}}}=\xi^{s}$, 
that is, $\mathcal{U}_{\xi^{s}} \in \Pi^{r}(\xi)$. Moreover, by Theorems 13 and 14(4), we have $\xi_{\mathcal{U}_{\xi}}^{l}=\xi^{s}$, that is, $\mathcal{U}_{\xi} \in \Pi^{l}\left(\xi^{s}\right)$.

(3) Let $B=\{e, b\} \subset E$ and $Y=\left\{h_{i} \mid i=\{1,2,3\}\right\}$ be given. Put $V, V \odot V \in S(Y \times Y, B)$ as

$\begin{array}{ccccccccc}V(e) & h_{1} & h_{2} & h_{3} & V(b) & h_{1} & h_{2} & h_{3} & \\ h_{1} & 1 & 0.6 & 0.5 & h_{1} & 1 & 0.5 & 0.3 & \\ h_{2} & 0.1 & 1 & 0.5 & h_{2} & 0.7 & 1 & 0.5 & \\ h_{3} & 0.4 & 0.6 & 1 & h_{3} & 0.6 & 0.6 & 1 & \\ (V \odot V)(e) & h_{1} & h_{2} & h_{3} & (V \odot V)(b) & h_{1} & h_{2} & h_{3} \\ h_{1} & 1 & 0.2 & 0 & h_{1} & 1 & 0 & 0 \\ h_{2} & 0 & 1 & 0 & h_{2} & 0.4 & 1 & 0 \\ h_{3} & 0 & 0.2 & 1 & h_{3} & 0.2 & 0.2 & 1\end{array}$

We define $\mathcal{U}_{Y}: S(Y \times Y, B) \rightarrow[0,1]$ as follows:

$$
\mathcal{U}_{Y}((U, A))= \begin{cases}1, & \text { if }(U, A)=\left(1_{Y \times Y}, B\right) \\ 0.6, & \operatorname{if}(U, A) \geq(V, B), \\ 0.3, & \text { if }(U, A) \geq(V \odot V, B),(U, A) \geq(V, B), \\ 0, & \text { otherwise. }\end{cases}
$$

Since $(V, B) \circ(V, B)=(V, B)$ and $(V \odot V, B) \circ(V \odot V, B)=(V \odot V, B), \mathcal{U}_{Y}$ is a soft $L$-fuzzy quasi-uniformity on $Y$.

From Theorem 13, we obtain a soft $L$-fuzzy topogenous order $\xi_{\mathcal{U}_{Y}}^{r}: S(Y, B) \times$ $S(Y, B) \rightarrow L$ as follows

$$
\xi_{\mathcal{U}_{Y}}((F, B),(G, B))= \begin{cases}1, & \text { if }\left[\left(1_{Y \times Y}, B\right)\right](F, B) \leq(G, B), \\ 0.6, & \text { if }[(V, B)](F, B) \leq(G, B), \\ & {[(V \odot V, B)](F, B) \leq[(V, B)](F, B),} \\ 0.3, & \text { if }[(V \odot V, B)](F, B) \leq(G, B) \\ 0, & \text { otherwise, }\end{cases}
$$

From Theorem 14, we obtain $\mathcal{U}_{\mathcal{\xi}_{\mathcal{U}_{Y}}^{r}}: S(Y \times Y, B) \rightarrow[0,1]$ as follows:

$$
\mathcal{U}_{\xi_{\mathcal{U}_{Y}}^{r}}((U, B))= \begin{cases}1, & \text { if }(U, B)=\left(1_{Y \times Y}, B\right) \\ 0.6, & \text { if }(U, B) \geq\left(U_{V_{F}, V_{F}}, B\right), \\ 0.3, & \text { if }(U, B) \geq\left(U_{(V \odot V)_{F},(V \odot V)_{F}}, B\right),(U, B) \geq(V, B), \\ 0, & \text { otherwise, }\end{cases}
$$

where $[(V, B)](F, B)=\left(V_{F}, B\right)$ and $[(V \odot V, B)](F, B)=\left((V \odot V)_{F}, B\right)$. Since

$$
\begin{aligned}
& \bigvee_{x \in X}(V(b)(x, y) \odot F(b)(x)) \rightarrow \bigvee_{x \in X}(V(b)(x, z) \odot F(b)(x)) \\
& \geq \bigwedge_{x \in X}((V(b)(x, y) \odot F(b)(x)) \rightarrow(V(b)(x, z) \odot F(b)(x))) \\
& \geq \bigwedge_{x \in X}(V(b)(x, y) \rightarrow V(b)(x, z)) \geq V(b)(y, z),
\end{aligned}
$$


$U_{V_{F}, V_{F}} \geq V$ and $U_{(V \odot V)_{F},(V \odot V)_{F}} \geq V \odot V$. Hence $\mathcal{U}_{\xi_{\mathcal{U}_{Y}}} \leq \mathcal{U}_{Y}$

\section{References}

[1] K.V. Babitha, J.J. Sunil, Soft set relations and functions, Compu. Math. Appl., 60 (2010), 1840-1849, doi: 10.1016/j.camwa.2010.07.014.

[2] N. Cag̈man, S. Karatas and S. Enginoglu, Soft topology, Comput. Math. Appl., 62 (1) (2011), 351-358. doi: 10.1016/j.camwa.2011.05.016.

[3] F. Feng, X. Liu, V.L. Fotea, Y.B. Jun, Soft sets and soft rough sets, Information Sciences, 181 (2011), 1125-1137, doi: 10.1016/j.ins.2010.11.004.

[4] P. Hájek, Metamathematices of Fuzzy Logic, Kluwer Academic Publishers, Dordrecht (1998), doi: 10.1007/978-94-011-5300-3.

[5] U. Höhle, S.E. Rodabaugh, Mathematics of Fuzzy Sets: Logic, Topology, and Measure Theory, The Handbooks of Fuzzy Sets Series 3, Kluwer Academic Publishers, Boston, 1999, doi: 10.1007/978-1-4615-5079-2.

[6] A.K. Katsaras, On fuzzy uniform spaces, J. Math. Anal. Appl., 101, 1984, 97-113. doi: 10.1016/0022-247x(84)90060-x.

[7] Y.C. Kim, Soft $L$-fuzzy preuniformities indued by soft $L$-fuzzy topogenous orders, (accepted to) Int. J. Pure and Applied Math.

[8] J.M. Ko, Y.C. Kim, Soft $L$-topologies and soft $L$-neighborhood systems, (accepted to) J. Math. Comput. Sci.

[9] J.M. Ko, Y.C. Kim, Soft $L$-uniformities and soft $L$-neighborhood systems, (accepted to) J. Math. Comput. Sci.

[10] J.M. Ko, Y.C. Kim, Soft $L$-fuzzy quasi-uniformities and soft $L$-fuzzy topogenous orders, (submit to) Int. J. of Pure and Applied Math.

[11] R. Lowen, Fuzzy uniform spaces, J. Math. Anal. Appl., 82 (1981), 370-385, doi: $10.1016 / 0022-247 x(81) 90202-x$.

[12] D. Molodtsov, Soft set theory, Comput. Math. Appl., 37 (1999), 19-31.

[13] Z. Pawlak, Rough sets, Int. J. Comput. Inf. Sci., 11 (1982), 341-356.

[14] Z. Pawlak, Rough probability, Bull. Pol. Acad. Sci. Math., 32 (1984), 607-615.

[15] M. Shabir and M. Naz, On soft topological spaces, Comput. Math. Appl.,61 (2011), 1786-1799, doi: 10.1016/j.camwa.2011.02.006.

[16] B. Tanay and M. B. Kandemir, Topological structure of fuzzy soft sets, Comput. Math. Appl., 61 (10) (2011), 2952-2957, doi: 10.1016/j.camwa.2011.03.056.

[17] $\mathrm{Hu}$ Zhao and Sheng-Gang Li, L-fuzzifying soft topological spaces and L-fuzzifying soft interior operators, Ann. Fuzzy Math. Inform., 5 (3) (2013), 493-503.

[18] Í. Zorlutuna, M. Akdag, W. K. Min and S. Atmaca, Remarks on soft topological spaces, Ann. Fuzzy Math. Inform., 3 (2) (2012), 171-185. 
